
cultures

Les cahiers de l'Acedle

15-1 | 2018

La conceptualisation grammaticale

\title{
Conceptualiser à partir d'un corpus oral : l'influence de la « doxa » grammaticale
}

Anick Giroud, Christian Surcouf et Malika Ben Harrat

\section{OpenEdition}

Journals

Édition électronique

URL : http://journals.openedition.org/rdlc/2703

DOI : $10.4000 /$ rdlc. 2703

ISSN : 1958-5772

Éditeur

ACEDLE

Référence électronique

Anick Giroud, Christian Surcouf et Malika Ben Harrat, « Conceptualiser à partir d'un corpus oral :

l'influence de la « doxa » grammaticale », Recherches en didactique des langues et des cultures [En ligne],

15-1 | 2018, mis en ligne le 02 janvier 2018, consulté le 01 mai 2019. URL : http://

journals.openedition.org/rdlc/2703; DOI : 10.4000/rdlc.2703

Ce document a été généré automatiquement le 1 mai 2019.

\section{c) (i) $९$}

Recherches en didactique des langues et des cultures is licensed under a Creative Commons AttributionNonCommercial-NoDerivatives 4.0 International License 


\title{
Conceptualiser à partir d'un corpus oral : l'influence de la « doxa » grammaticale
}

\author{
Anick Giroud, Christian Surcouf et Malika Ben Harrat
}

\section{Introduction}

1 Quand des efforts sont faits pour intégrer dans l'enseignement les savoirs issus des recherches récentes en linguistique (voir par ex. Bertrand \& Schaffner, 2013), se pose le problème de l'inadéquation de ces nouveaux savoirs aux représentations métalinguistiques déjà en place chez l'apprenant, notamment du fait des manuels et de la pratique enseignante (Beacco, 2015). Dans le cas d'une activité de "conceptualisation » (voir Besse, 1974; Beacco, 2010), comment ces représentations de la "grammaire ordinaire » (ou doxa grammaticale selon Beacco, 2015 : 24), fondées sur les explications des manuels et des grammaires interviennent-elles dans le processus de réflexion ? En milieu d'apprentissage homoglotte, on s'attendrait à ce que l'apprenant construise sa grammaire intériorisée (Besse \& Porquier, 1984) sur la base des données orales issues de son environnement. Que se passe-t-il lors d'une tâche de conceptualisation en classe à partir d'un corpus de données orales? Comment les sujets appréhendent-ils le matériau sonore ? Comment s'y prennent-ils pour conceptualiser une règle grammaticale décrivant le fonctionnement oral de la langue? Pour explorer ces questions, on a observé les interactions d'apprenants engagés dans une telle tâche de verbalisation de la formation du Futur (dit « simple » dans la tradition grammaticale).

2 L'expérimentation, menée en contexte universitaire et présentée comme tâche d'expression orale, a concerné 20 étudiants de FLE (B1). Répartis en 5 groupes de 4, de langues maternelles différentes, ils avaient 40 minutes pour trouver une ou plusieurs règles à partir d'un corpus oral de 20 exemples, présentés sous la forme de fichiers mp3, disponibles sur la plateforme Moodle du cours ${ }^{1}$. La consigne était la suivante : 
Vous allez écouter une série d'énoncés exprimant le futur avec 20 verbes différents. En groupe, discutez afin de trouver la ou les règles pour expliquer la formation des verbes au futur simple. Vérifiez que votre explication fonctionne bien avec les verbes que vous avez entendus. Quand vous êtes d'accord, formulez clairement votre règle et appelez les enseignantes : l'exercice est terminé.

3 La démarche a consisté à analyser les verbalisations donnant accès aux représentations métalinguistiques et métacognitives mobilisées (voir Gombert, 1990 ; Aguerre, 2010 : 68). Avant de commenter nos résultats, nous présentons les problèmes posés par le discours de la grammaire ordinaire sur la formation du Futur.

\section{Aperçu des écueils du recours à l'orthographe dans l'explication pédagogique}

4 Si l'on admet comme incontournable le «caractère vocal » de la langue (Martinet, 2008: 32), alors " la conjugaison orale doit être considérée comme la conjugaison de base » (Pinchon \& Couté, 1981: 13). Un tel positionnement se justifie par la définition du morphème : «entité linguistique réunissant à la fois un signifiant et un signifié » (Neveu, 2004 : 196). Toutefois, dans sa pratique ordinaire, le linguiste se retrouve confronté au paradoxe épistémologique consistant à recourir à l'écrit (silencieux) pour décrire l'oral, problème d'autant plus aigu en français que le rapport graphème-phonème de l'orthographe est complexe (Fayol \& Jaffré, 2014: 23). Prenons l'exemple de la décomposition morphologique sur une base orthographique du verbe "grandir». Graphiquement, "grandir » se décompose sans difficulté apparente en "grand + ir ". Alors que «-ir », lu /iь/, renvoie au morphème de l'Infinitif, « grand» - que tout locuteur natif lira /gBã - parait renvoyer à l'adjectif masculin. Cependant, il s'agit là d'une illusion générée par l'orthographe et sa lecture: /gБãdiь/ se décompose en effet en /gвãd+iь/, dérivation de l'adjectif « féminin »/gвãd/, comme on l'observe ailleurs avec /blãf+iв/, / bьуп+iь/, etc.

5 En d'autres termes, l'orthographe française est loin de constituer l'outil idéal d'analyse et de description morphologique (voir Surcouf, 2011). Pourtant depuis plusieurs décennies en dépit des propositions déjà anciennes de Csécsy (1968) ou de Pinchon \& Couté (1981) les concepteurs de grammaires et de manuels de FLE recourent presque exclusivement à l'orthographe pour leurs descriptions. Se pose alors la question de savoir ce que peut faire l'apprenant d'une explication orthographique comme le Futur est un temps simple (radical + terminaison). Le radical est l'infinitif. Tous les verbes ont les mêmes terminaisons. commencer $>$ je commencerai ; partir > je partirai (Akyüz et al., 2015 : 132). Si dans le cas de «partir», une telle règle fonctionne tant graphiquement qu'oralement, permettant de lire /pabtib+e/ la concaténation orthographique " partir + ai », tel n'est pas le cas pour « commencer + ai ». L'Infinitif se lisant /komãse/, l'ajout de /e/ aboutirait logiquement à /komãsee/. Bien qu'il soit probable que l'apprenant ait suffisamment intégré le fonctionnement de l'orthographe française pour ne pas lire /komãsee/, il lui sera en revanche difficile d'éviter la lecture erronée /komãseьe/, où le segment en gras reproduit la prononciation de l'Infinitif, censé entrer dans la formation de ce temps.

6 En somme, l'orthographe masque en partie le fonctionnement ciblé par l'explication pédagogique, et l'apprenant de FLE se voit confronté à une triple difficulté : il ne maitrise 
ni les formes orales de la conjugaison, ni l'orthographe tant en écriture qu'en lecture ${ }^{2}$. Pourtant comme le relève Goes (2015 : 65), le Futur est présenté de façon assez uniforme par les grammaires pédagogiques, et les explications s'inscrivent dans une longue tradition orthographique, érigeant l'Infinitif comme base de formation: Du présent de l'infinitif se forme le futur de l'indicatif [...], en changeant $r$ ou re en rai [...], comme aimer, j'aimerai, [...]; rendre, je rendrai (Bescherelle, 1858 : LI). Qu'en est-il dans les manuels et les grammaires de FLE actuels?

\section{La formation du Futur dans quelques manuels et grammaires de FLE}

7 Ce court examen offre un aperçu des explications de 20 ouvrages de FLE, publiés entre 1990 et $2015^{3}$ :

Tableau 1 - Répartition des manuels et grammaires analysés

\begin{tabular}{|l|l|l|l|}
\hline & $1990-1999$ & $2000-2009$ & $2010-2015$ \\
\hline Manuels & 2 & 5 & 4 \\
\hline Grammaires & 2 & 1 & 6 \\
\hline
\end{tabular}

Sur les 11 manuels, 4 ne fournissent aucune explicitation claire de la formation du Futur, se contentant de tableaux (Flumian et al., $2004: 115)$ ou d'exemples (Kaneman-Pougatch et al., 1997 : 116), dans lesquels la présentation orthographique de type «j'aimerai », « il finira" et la spécification des terminaisons "-ai», "-as", etc. semblent inciter l'apprenant à considérer l'Infinitif comme radical ${ }^{4}$.

Dans les 7 autres manuels, l'Infinitif est explicitement signalé comme base de formation du Futur, soit lapidairement comme dans Saison 1 : verbe à l'infinitif + - ai, - as, - $a$, - ons, -ez, ont (Cocton et al., 2014 : 196), soit en détail :

Le futur se forme en général à partir de l'infinitif :

- verbes terminés par -ER ou -IR

$>$ terminaisons en - $a i$, - $a$ s, $-a$, -ons, -ez, -ont

- verbes terminés par -RE (vendre, prendre, lire, écrire...)

$>$ on supprime le $-e$ final et on ajoute les terminaisons. (Chahi et al., 2015 :

202)

Parmi les 9 grammaires analysées, 2 ne proposent que des tableaux (Abry \& Chalaron, 2014 : 230), 6 font explicitement remonter la formation du Futur à l'Infinitif, à l'instar de Poisson-Quinton (2014: 70) : Quand les verbes se terminent par - $[$ [...] on prend l'infinitif + -ai, $a s,-a$, -ons, -ez, -ont, et pour les autres verbes, il est préconisé de supprimer le «e » de l'Infinitif et d'ajouter lesdites terminaisons. Si ces 8 premiers ouvrages arborent les mêmes désinences orthographiques (-ai, -as, etc.), la Grammaire utile du français propose une solution avec 3 types de terminaisons :

Verbes en er (sauf aller et envoyer). Pour tous ces verbes (plus de 9000), la formation du futur est simple : verbe au présent avec je + rai, ras, ra, rons, rez, ront. [...] 
Verbes en ir qui se conjuguent sur le modèle des verbes en er. À l'exception des verbes cueillir, accueillir, recueillir, tous font leur futur en rai, ras, ra, etc. (Bérard \& Lavenne, $1991: 283$ )

La décomposition orthographique est : «j' offri rai » «j' ouvri rai ». Quant à " cueillir » et ses dérivés, ils font leur futur en -erai soit je cueill erai (ibid.) et la formation du futur des autres verbes en "ir » est: Infinitif + ai, as, a, ons, ez, ont » (Bérard \& Lavenne, 1991 : 284). Enfin, pour les verbes à Infinitif en « re », ils font leur futur de la façon suivante : Infinitif-e + ai, as, a, ons, ez, ent (ibid.).

11 En somme, à l'exception de Bérard \& Lavenne (1991), les présentations fournies par les concepteurs de manuels ou de grammaires de FLE reposent essentiellement sur l'agencement orthographique de l'Infinitif et des terminaisons «-ai », "-as ", etc. Pourtant, comme l'illustre la tentative de ces deux auteurs, la question est loin d'être aussi simple que la tradition le laisse supposer, et le Futur constitue en définitive le temps le plus complexe (Pinchon \& Couté, 1981 : 80). Que nous révèle l'analyse des 6500 verbes du Petit Robert à cet égard?

\section{La description linguistique des 6500 verbes du Petit Robert}

Comme notre aperçu des explications des manuels le laissait entrevoir, deux séries de désinences sont possibles : / $\mathrm{Be} /$, /ва/, /ьõ/ ou /e/, /a/, /õ/, chacune se répercutant sur la nature du radical. Ces alternatives admises, le Futur pourrait se former sur le Pr(ésent) et/ou l'Inf(initif). Quelles sont alors les configurations envisageables ( 0 : impossible, 1 : possible)?

Tableau 2 - Formation du futur : Configurations possibles

\begin{tabular}{|c|c|c|c|c|}
\hline & pr & inf & Analyse & Exemple de décomposition \\
\hline a) & 1 & 0 & univoque & $/ \operatorname{lav}+\mathrm{Ba} /$ mais pas /*lavb $+\mathrm{a} /$ \\
\hline b) & 0 & 1 & univoque & $/$ kuds $+\mathrm{a} /$ mais pas /*kud+Ba/ \\
\hline c) & 0 & 0 & impossible & /vudьa/, ni /*vud+вa/ ni /*vudь+a/ \\
\hline d) & 1 & 1 & ambigüe & $/ \mathrm{li}+\mathrm{Ba} /$ ou $/ \mathrm{lis}+\mathrm{a} /$ \\
\hline
\end{tabular}

Les configurations a) et b) sont univoques dans la mesure où en a) /lavba/ s'analyse exclusivement en /lav+Ba/, où /lav/ renvoie au radical du Présent, et de même en b) pour /kudsa/ analysable en $/ \mathrm{kud}_{\mathrm{H}}+\mathrm{a} /$, où $/ \mathrm{kud} \mathrm{s} /$ représente l'Infinitif. La décomposition fait ici apparaitre une variation possible dans les désinences. En c), /vudba/ est indécomposable quelle que soit la flexion choisie car ni /*vud/ ni /*vuds/ n'existent ailleurs dans le paradigme de «vouloir ». Enfin en d), /lisa/ peut donner lieu à deux décompositions concurrentes, l'une fondée sur le radical singulier du Présent /li/+/ba/, l'autre sur l'Infinitif /liк/+/a/. En raison de la diversité de composition du Présent, en dehors des supplétions ${ }^{5}$ telles que /іва/, /ова/, etc., l'analyse exhaustive des 6500 verbes du Petit Robert ${ }^{6}$ débouche sur les 14 configurations du tableau 1. Les cases en nuances de gris indiquent le ou les radicaux servant à la formation du Futur. Par exemple, en 1, les 
verbes à Infinitif en «-er» et quelques-uns en " -ir» (« courir») se construisent sur le

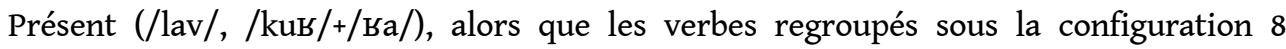
peuvent se décomposer de deux manières : soit sur le radical pluriel du Présent (/viv+Ba/ ), soit sur l'Infinitif (/vivi $+a /)$. Comme le montrent les pourcentages en gras de la colonne de droite, la formation sur un radical du Présent est très largement majoritaire,

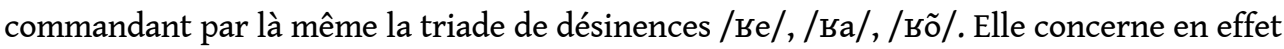
$90,8 \%$ des verbes $(1,5,9,14)$.

Tableau 3 - Les quatorze configurations de la formation du Futur (hors supplétion)

\begin{tabular}{|c|c|c|c|c|c|c|c|c|}
\hline & \multicolumn{3}{|l|}{ Pr } & \multirow{2}{*}{ Inf } & \multirow{2}{*}{ Remarques } & \multirow{2}{*}{ Exemples } & \multirow{2}{*}{ n } & \multirow{2}{*}{$\%$} \\
\hline & 1-3 & 4-5 & 6 & & & & & \\
\hline 1. & $\mathrm{xx}$ & $\mathrm{xx}$ & $\mathrm{XX}$ & & inf en /e/ \& qlq. /іь/ & $\operatorname{lav} \mathbf{s} \mathbf{a}, \operatorname{ku} \mathbf{B} \mathbf{a}$ & 5541 & 86,4 \\
\hline 2. & $\mathrm{xx}$ & $\mathrm{XX}$ & $\mathrm{xX}$ & $\mathrm{X}$ & inf en /ув/ \& qlq. /ів/ & kõklyва, fціва, віва & 9 & 0,1 \\
\hline 3. & & & & $\mathrm{x}$ & inf en /сСів/, /jiв/ & uvвіва, аsајіва & 13 & 0,2 \\
\hline 4. & $\mathrm{xx}$ & & $\mathrm{xX}$ & $\mathrm{X}$ & inf en /waь/, /єь/ & kьшава, арstьєьа & 17 & 0,3 \\
\hline 5. & $\mathrm{xx}$ & & & & pr en /ع/-/ə/, /wa.j/ & $\operatorname{l\varepsilon v\mathbf {s}} \mathbf{a}$, netwa $\mathbf{a}$ & 262 & 4,1 \\
\hline 6. & & $\mathrm{XX}$ & & & mourir,-quérir & $\operatorname{mus\mathbf {B}} \mathbf{a}, \operatorname{ak\varepsilon } \mathbf{6} \mathbf{a}$ & 6 & 0,1 \\
\hline 7. & $\mathrm{xx}$ & & & $\mathrm{x}$ & inf en /ів/, /عь/ & fіпіьа, liьа, plєьа & 374 & 5,8 \\
\hline 8. & & $\mathrm{XX}$ & $\mathrm{xX}$ & $\mathrm{X}$ & inf en /Сь/ & vãdьа, vivьа, batьа, рєьdьа & 88 & 1,4 \\
\hline 9. & & & $\mathrm{xx}$ & & pleuvoir & plœv $\mathbf{B} \mathbf{a}$ & 2 & 0 \\
\hline 10. & & & & $\mathrm{X}$ & inf en /ів/, /VСь/ & sãtiвa, kudьa, nєtьa & 45 & 0,7 \\
\hline 11. & & & & $\mathrm{x}$ & inf en -soudre, -indre & веzudьa, pг̃dьa & 33 & 0,5 \\
\hline 12. & $\mathrm{xx}$ & & & $\mathrm{X}$ & boire & bшава & 1 & 0 \\
\hline 13. & & & & $\mathrm{x}$ & -prendre & рьãdьa & 12 & 0,2 \\
\hline 14. & & $\mathrm{XX}$ & & & -evoir, -ouvoir & 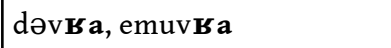 & 11 & 0,2 \\
\hline tot & & & & & & & 6414 & $100 \%$ \\
\hline
\end{tabular}

Le numéro renvoie aux personnes : 1 pour je, 2 pour tu, etc. Les cases comportant $X$ correspondent à I'Infinitif, celles comportant XX au radical du Présent usité pour le Futur.

$\mathrm{C}$ et $\mathrm{V}$, pour consonne et voyelle, qlq. pour quelques

Ces statistiques montrent que les explications pédagogiques fondant la formation $\mathrm{du}$ Futur sur l'Infinitif + /e/, /a/, /õ/ s'avèrent injustifiées puisqu'elles ne concernent que $1,6 \%$ des verbes de manière univoque $(3,10,11,13)$, ou éventuellement $9,2 \%$ si l'on inclut les $7,6 \%$ des configurations à décomposition ambigüe $(2,4,7,8,12)$. 


\section{Le corpus oral des verbes}

Pour le corpus oral des formes du Futur soumis à l'écoute des apprenants, l'un des auteurs a enregistré les 20 verbes retenus, systématiquement conjugués avec les trois formes en /ве/, /ва/, /ьо̃/, et intégrés dans des énoncés ${ }^{7}$ articulés de la manière la plus naturelle possible malgré l'artificialité engendrée par l'objectif de l'expérimentation ${ }^{8}$. Ainsi pour le verbe "mettre", les apprenants entendaient l'énoncé Demain, tu mettras quoi? Moi, je mettrai mon pantalon rouge, et les enfants, ils mettront quoi pour pas avoir trop chaud? En dépit de sa taille réduite, le corpus des verbes s'efforce de refléter les proportions et le fonctionnement mis en évidence dans le tableau 1. Six types de construction ont été retenus. Comme le synthétise le tableau 2, la règle de formation sur

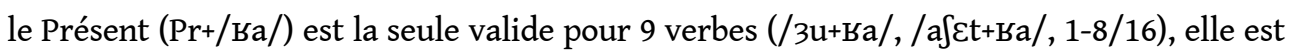
possible pour 7 verbes (9-15), mais en concurrence avec l'Infinitif (/li+ba/ ou /lib+a/). Elle est en revanche impossible pour 4 verbes (17-20). Pour 17-18, seul l'Infinitif fonctionne, alors que 19-20 requerront la mémorisation d'un radical spécifique.

Tableau 4 - Les 20 verbes sélectionnés pour le corpus oral

\begin{tabular}{|l|l|l|}
\hline Verbes retenus & Radical & $\begin{array}{l}\mathrm{Pr}+/ \\
\mathbf{6} \text { a/ ? }\end{array}$ \\
\hline $\begin{array}{l}\text { 1) jouer, 2) étudier, 3) manger, 4) apporter, 5) écouter, 6) arriver, } \\
\text { 7) demander, 8) habiter }\end{array}$ & $\operatorname{Pr} 1-6$ & oui \\
\hline 9) lire, 10) finir, 11) écrire & $\begin{array}{l}\operatorname{Pr} 1-3 \quad \text { ou } \\
\text { Inf }\end{array}$ & possible \\
\hline 12) mettre, 13) attendre, 14) vendre, 15) répondre & $\begin{array}{l}\operatorname{Pr} 4-6 \quad \text { ou } \\
\text { Inf }\end{array}$ & possible \\
\hline 16) acheter & Pr1-3/6 & oui \\
\hline 17) dormir, 18) sortir & Inf & non \\
\hline 19) venir, 20) pouvoir & Supplétion & non \\
\hline
\end{tabular}

\section{Résultats}

Dans l'ensemble, la tâche a suscité la réflexion sur la langue, et les commentaires métalinguistiques et métacognitifs sont abondants, permettant dès lors de mieux cerner les stratégies employées et d'accéder aux représentations des apprenants. Dans le cadre limité de cet article, on s'intéressera uniquement à la manière dont les étudiants ont appréhendé ce corpus oral, et aux difficultés qu'ils ont rencontrées dans l'accès au sens des énoncés oraux, et dans la formulation d'une règle morphologique. 


\section{Compréhension de la tâche}

17 Dans tous les groupes, on constate une difficulté à saisir le sens de l'activité (objectifs, nature du problème à résoudre, actions à mener, etc.). Par son caractère inhabituel réflexion métalinguistique à partir d'un corpus «oral "-, la consigne déstabilise les apprenants et donne lieu à des discussions et des demandes d'aide. Ces commentaires s'orientent dans trois directions: la finalité de la tâche («quoi faire ? »), la manière de procéder (« comment faire? ?) et la légitimité de l’activité (« pour quoi faire?»).

\section{Le « quoi faire »}

Dans tous les groupes, le problème de la compréhension de la consigne est verbalisé :

$2 \mathrm{Am}^{9}:$ tu comprends ce que on doit faire?

Les demandes d'aide s'adressent soit aux autres membres du groupe, soit aux enseignantes, parfois à un autre groupe. Un apprenant au moins dans chaque groupe fournit des explications en reformulant la consigne :

3Su : on doit trouver les règles de comment se fait le futur simple 2Am : normalement si je bien compris on doit régler la norme pour formuler le futur simple

Ils verbalisent ainsi leurs représentations de la tâche de conceptualisation. La question de l'écrit apparait d'emblée dans tous les groupes:

3An : oui oui on doit écrire tous les verbes et après trouver on peut peut-être $1 \mathrm{Mg}$ : on doit prendre tous les verbes parce que à la fin on doit écrire la règle pour construire le futur

\section{Le « comment faire »}

On trouve des commentaires métacognitifs sur la manière de faire :

3Di : on on va discuter ensemble pour chaque verbe?

3Su: je pense que on écoute tout parce que avec un seul tu peux pas trouver rien non?

Ces verbalisations nous donnent accès aux stratégies mobilisées dans l'activité de conceptualisation. Elles montrent la capacité qu'a l'apprenant à prendre du recul sur l'activité tout en réfléchissant aux opérations à effectuer et à leur organisation. Du point de vue didactique, cette focalisation des échanges sur les actions à mettre en œuvre est intéressante car l'explicitation métacognitive durant l'interaction dans les groupes est susceptible de permettre aux étudiants en difficulté de prendre conscience de cet aspect de l'apprentissage (Griggs, 2002). Le commentaire de 3Su explicite le principe même sur lequel repose l'activité de conceptualisation: c'est la mise en relation des éléments observés qui permet d'inférer la règle, grâce aux processus de généralisation et de discrimination.

Classifier les phénomènes est un autre exemple de stratégie à mettre en œuvre dans l'action de conceptualiser : 
3Di : pas besoin de discuter forcément de les terminaisons [...] on doit eh faire classification accordé eh concernant par rapport à verbe suivant le 20 verbes ici

\section{Le « pour quoi faire »}

d'emblée son rejet de l'activité :

7Zh : mais il y a beaucoup de exemples

Cette réaction conditionne sa posture jusqu'à la fin :

$7 Z h$ : je pense que c'est c'est nul hein [...] oui ces ces vingt exemples c'est nul

Elle participe peu aux échanges, et ne produit jamais d'interventions initiatives. Sollicitée par ses partenaires, elle répond en raisonnant sur le fonctionnement « écrit » du futur :

$$
\text { on /efase/ le /e/ et on /azute/ le /a/ /i/ }{ }^{10}
$$

En définitive, la tâche de conceptualisation n'avait aucun sens pour elle.

28

2. Sa explicite également son incompréhension de la tâche dès le début de l'interaction :

3Sa : cherche cherche eh pas je comprends pas ils cherchent un mot il faut je dois chercher ou quoi?

Il exprime sa frustration :

3Sa : juste discuter?

Quand son partenaire lui répond: 
3Di : on écoute il n'y a pas besoin de d'écrire

3Sa manifeste une certaine déstabilisation qu'il cherche à éviter en recourant à l'écrit :

3Sa : mais on peut hein elle a dit que on peut écrire si on veut

Son intervention porte les traces du système de valeurs déterminant son comportement dans l'apprentissage. Comme 7Zh, sa réflexion se base sur ses connaissances à l'écrit, comme l'illustre ce commentaire sur le verbe mettre : «on enlève le /ə/ », renvoyant en l'occurrence à la dimension graphique (voir 6.2). On verra en 8.2 comment cette représentation le pousse à produire des formes en contradiction avec les données du corpus.

Signalons par ailleurs que tous les apprenants « réticents » enfreignent à un moment ou un autre l'interdiction de recourir à Internet : ils consultent des ressources en ligne qui ne leur seront d'aucun secours puisque fondées sur l'écrit. Bien que ces ressources ne permettent pas de résoudre le problème posé, elles rassurent les apprenants déstabilisés par une consigne et un corpus inhabituels. Si l'usage d'Internet parait désormais naturel, l'exercice de conceptualisation grammaticale, apparu dans les années 1970, visait l'autoconstruction autonome et idiosyncrasique de la règle ciblée. Cette donnée doit donc être prise en compte pour un renouvellement de la démarche.

\section{Aspect motivationnel}

30 L'incompréhension de l'objectif est de plus associée à divers comportements : repli sur soi (absence d'initiatives dans le groupe, peu d'interventions), stylo constamment à la main, décalage temporel par rapport aux autres, etc. On retrouve ici des observables classiques du degré de motivation des apprenants. Ne pas comprendre l'enjeu entraine une baisse de motivation chez les « réticents " (Sa est l'exception). De plus, il est logique que le fait d'être convaincu de déjà connaitre la réponse diminue la volonté de s'investir dans la tâche, mais ce n'est pas toujours le cas.

31 À l'opposé, les apprenants le plus aptes à verbaliser les objectifs et les stratégies souhaitables ont souvent un comportement de meneur de l'interaction, sollicitant les autres, prenant des initiatives, émettant des hypothèses sur la règle et développant une forte activité d'étayage.

\section{Appréhension du matériau sonore}

Les caractéristiques des enregistrements ont entrainé des difficultés dans la compréhension des énoncés : 1) le corpus est discontinu ;2) les énoncés apparaissent sans contexte de production ; 3) la prononciation, l'intonation et la morphosyntaxe se veulent proches de "l'authentique». Aussi pour l'étudiant ayant un faible niveau de compréhension orale, l'exercice de conceptualisation devient difficile car il met en jeu une triple contrainte: comprendre les énoncés oraux en temps réel (même s'il est possible de les écouter autant de fois qu'il le désire), identifier le verbe conjugué et les trois terminaisons /ве/, /ва/, /ьõ/, et en déduire la formation du futur simple.

Selon les groupes, l'attention se porte plus ou moins fréquemment sur la compréhension orale à partir des difficultés vécues en temps réel : je comprends rien (3Di). La comparaison entre les groupes montre que la saisie du matériau sonore détermine le déroulement du 
processus de conceptualisation : dans G7, par exemple, un locuteur arabophone tunisien aide les autres à comprendre le sens des items. De ce fait, les problèmes rencontrés sont vite résolus et le groupe passe peu de temps à négocier le sens. En revanche, dans G3, où la majorité a un niveau de compréhension faible, ce problème ralentit le déroulement de l'activité en raison d'un temps d'écoute plus long mais aussi d'un nombre d'échanges accru de co-construction de la signification de l'énoncé par la reformulation collective (68 tours de parole pour comprendre l'item 1). Ces moments de collaboration sont intéressants du point de vue de l'apprentissage. Ils indiquent des mouvements d'étayage où les rôles peuvent s'intervertir, ponctués de retours à l'écoute pour vérifier les hypothèses mutuelles.

\section{Construction et formulation de la règle}

Le recours à l'écrit, constant chez les apprenants, remplit trois fonctions pour :

comprendre: stratégie habituelle profondément ancrée dans les pratiques d'apprentissage, comme 3Sa en fait la preuve, en s'adressant d'abord à l'enseignante :

3Sa : mais on peut écrire quelque chose non? qu'est-ce que on entend on peut écrire non madame

puis à un pair :

mais on peut hein elle a dit que on peut écrire si on veut

- se souvenir : approprié pour mémoriser des données orales ${ }^{11}$;

37 - expliquer la règle : a) l'écrit sert d'appui à la discussion ; b) le recours à l'orthographe est systématique pour la construction de la règle, ce qui mène souvent à une impasse.

Émerge alors une tension entre l'écrit et les données orales, rendant plus délicate la formulation d'une règle satisfaisante.

\section{Tension entre les dimensions écrites et orales}

On a vu en que, le discours de la grammaire ordinaire abordait la description de la morphologie orale par le biais de l'écrit, seule norme envisagée. Il en est de même pour les savoirs grammaticaux antérieurs sur lesquels s'appuient les apprenants. Cette prégnance de l'écrit conditionne l'activité réflexive. Tout se passe comme si le savoir théorique déjà engrangé venait faire écran à la saisie du matériau sonore. Par exemple pour le verbe «mettre », plusieurs groupes mentionnent le fait qu'il suffit d'enlever le / ə/, explication également récurrente dans les manuels et grammaire de FLE. La conceptualisation du phénomène s'avère alors problématique puisqu'il s'agit de désigner une forme orale /mets/, où rien ne correspond à ce /ə/. Il n'y a donc rien à « enlever » ou « jeter ».

Le recours à l'écrit, apparemment indispensable à la plupart des étudiants dans leur discours explicatif, engendre donc des ambigüités constantes, repérables notamment quand l'un des participants, comme $2 \mathrm{Al}$, est attentif à dégager une règle fonctionnant à l'oral. Le contact entre les deux modes oral/écrit se régule de manière explicite :

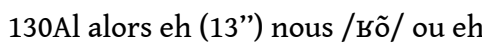

131Am nous quoi? 
132Al nous (hésite) /mعtıõ/?

133 Am nous /mєteвõ/

$134 \mathrm{Al}$ nous /metrõ/ c'est fini?

135Am ça s'écrit comment ? met $t$ ? avec double $t$ ou pas ? non?

$136 \mathrm{Al}$ (soupir) je sais pas ta question (rires)

\section{Al en 130-132-134 cherche à appliquer une règle opératoire pour l'oral, alors que $2 \mathrm{Am}$ raisonne à partir de l'écrit et précise ses sollicitations. Ce double mode de fonctionnement est présent dans tous les groupes et constitue un aspect important de la tâche. Il est aussi interne : 1Sd par exemple juxtapose les deux modes de raisonnement : \\ on fait toujours comme ça on ajoute /a/ /i/ /Зәравləье/ pour je /a/ /عs/ pour tu}

Il ajoute :

et puis tu dois faire autre chose tu dois prendre la conjugaison du présent ça veut dire par exemple pour le verbe acheter il faut dire /3afet/ [...] tu ajoutes $/ \varepsilon \boldsymbol{\varepsilon b} /[. .$.$] et puis tu ajoutes la terminaison$

\section{Imbrication étroite dans le discours}

Finalement, les discussions menées au sein des groupes en vue de formuler la règle consistent en un mélange de données orales et écrites. On assiste à la co-construction d'hypothèses hybrides comme dans G3 et G1 qui produisent les mêmes erreurs à partir du même raisonnement pour le verbe " jouer ». 3Sa écrit et explique simultanément : infinitif est/Zue/, montre ses notes et entraine l'échange suivant:

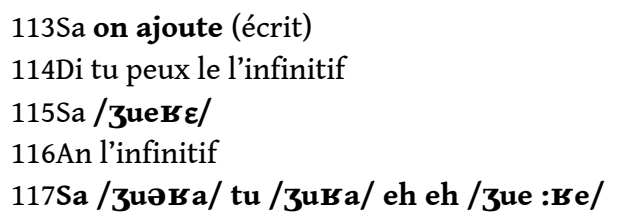

L'apprenant oralise ici des formes écrites issues de son application de la règle, en contradiction avec ce qu'il entend. Les hypothèses basées sur la règle traditionnelle (construction à partir de l'Infinitif) sont confrontées à la réalité de la production orale (les données du corpus). D'où les innombrables variations émises en collaboration: / зиьо̃/, /зивје/, /зиве/, /Зиево̃/, /Зиәьо̃/, /ә/-/ьо̃/, /Зиевє/. Pоur 3Sа, le matériau sonore vient perturber plutôt que modifier sa représentation de la formation du Futur. La multiplication des formes proposées semble indiquer de gros efforts sur le plan cognitif, les opérations activées sont ralenties (perception du matériau sonore, rappel de savoirs théoriques, oralisation des formes graphiques, gestion de l'interaction, etc.). 1Md produit le même raisonnement à partir de l'Infinitif :

/зшеке/ /зшева/ /зшева/ oui euh /зu//зше/-/во̃//зшеве/ et /зшеьо̃/ alors le radical c'est toujours /3we/

Elle aussi est en train d'écrire et de lire, et visuellement, "jouera » est le produit de « jouer + a ». Ainsi, privilégier la graphi ${ }^{12}$ aboutit la plupart du temps à un résultat nonconforme à la langue cible orale.

Recherches en didactique des langues et des cultures, 15-1 | 2018 


\section{Conclusion} tâche de conceptualisation grammaticale, pour essayer de comprendre comment les représentations prégnantes du discours normatif officiel déterminent non seulement le produit de l'activité, mais aussi sans doute son processus. œuvre sont verbalisées et discutées. Elles montrent comment les apprenants peinent à coconstruire des hypothèses sur la règle, qui s'avèrent le plus souvent non-conformes à la langue-cible. Le problème parait double : a) le discours normatif sur la formation du futur échoue à décrire la réalité du phénomène dans son ensemble ; b) l'explicitation des règles passe toujours par l'écrit, ce qui perturbe la conceptualisation du phénomène oral.

La tension sous-jacente entre l'écrit et l'oral dans les tentatives de formulation de la règle-cible ne permet pas de construire une "règle-action » opératoire. Trois apprenants seulement se sont appuyés sur les données orales et ont réussi à verbaliser leur fonctionnement. La prise en compte de l'ensemble des résultats de l'expérience devrait permettre de mieux cerner les conditions de réussite.

Il semble ainsi que la tâche de conceptualisation doit être pensée en fonction du « déjà là » et du «toujours là ", c'est-à-dire des savoirs déclaratifs métalinguistiques présents antérieurement chez les apprenants ou circulant dans le discours grammatical ordinaire disponible en ligne et dans les ouvrages de référence utilisés par les apprenants (principalement Guédon et al., 2013). Ces savoirs monopolisent la réflexion métalinguistique et constituent un obstacle à l'activité cognitive de conceptualisation visée par la tâche. Ils ont aussi pour effet de réduire le degré de motivation à se prêter à l'exercice. Terminons par l'exemple édifiant du commentaire métacognitif de 5Se (groupe des « réticents ») à la $19^{\mathrm{e}}$ minute :

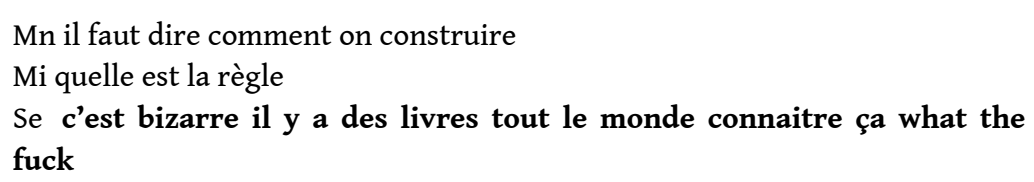

Beaucoup de questions restent en suspens. Comment favoriser une prise en compte réelle des données orales des corpus présentés? Comment aider les apprenants à mieux généraliser à partir d'exemples oraux ? Notre expérimentation n'est pas représentative d'une activité de classe (durée, enregistrement, etc.), mais un objectif pédagogique a été atteint : la séance a favorisé l'activation d'opérations réflexives et la confrontation des modes de représentation (écrit/oral). Enfin, on peut se demander dans quelle mesure les tâches collaboratives de conceptualisation grammaticale, qui génèrent une intense activité métalinguistique et métacognitive, pourraient jouer un rôle de déconstruction des représentations basées sur la norme écrite. 


\section{BIBLIOGRAPHIE}

Abry, D. \& Chalaron, M.-L. (2014). La grammaire des premiers temps A1-A2. Grenoble : Presses universitaires de Grenoble.

Aguerre, S. (2010). « Grammaire de l'apprenant et explication. Quand apprendre, c'est se construire des représentations, à partir de corpus et d'instruments ». Enseigner les structures langagières en FLE. Bruxelles : PIE Peter Lang. pp. 65-76.

Akyüz, A. ; Bazelle-Shahmaei, B. ; Bonenfant, J. \& Orne-Gliemann, M.-F. (2015). Grammaire du françaisA1-B1. Paris : Hachette.

Beacco, J.-C. (2010). La didactique de la grammaire dans l'enseignement du français et des langues. Paris : Didier.

Beacco, J.-C. (2015). « La grammaire : un long fleuve tranquille?». In Defays, J.-M. (dir.). Transversalités - 20 ans de FLES - Faits et gestes de la didactique du Français Langue Étrangère et Seconde de 1995 à 2015, vol. 1. pp. 15-27.

Bérard, É. \& Lavenne, C. (1991). Modes d'emploi. Grammaire utile du français. Paris : Didier.

Bérard, É., Canier, Y. \& Lavenne, C. (1996). Tempo 1. Méthode de français. Paris : Didier/Hatier.

Bertrand, O. \& Schaffner, I. (dir.) (2013). Enseigner la grammaire. Palaiseau : Éditions de l'École polytechnique.

Bescherelle, L.-N. (1858). Dictionnaire usuel de tous les verbes français tant réguliers qu'irréguliers entièrement conjugués. Tome premier : A-E. Paris : Garnier.

Besse, H. (1974). « Les exercices de conceptualisation ou la réflexion grammaticale au niveau 2 ». Voix et images du Crédif 2. pp. 38-44.

Besse, H. \& Porquier, R. (1984). Grammaires et didactique des langues. Paris : Hatier.

Blanche-Benveniste, C. (2003). La langue parlée. In Yaguello, M. (Ed.). Le Grand Livre de la Langue française. Paris : Seuil. pp. 317-344.

Chahi, F., Huor, C., Malorey, C., Marchandeau, C., Pruvost, N., Miras, G., Poisson-Quinton, S. \& Delannoy, G. (2015). Entre Nous 2 - A2. Méthode de français. Paris : Éditions Maison des langues.

Cocton, M.-N. ; Heu, E. ; Alcaraz, M. ; Dupleix, D. \& Ripaud, D. (2014). Saison 1 - A1-A2. Méthode de français. Paris : Didier.

Csécsy, M. (1968). De la linguistique à la pédagogie. Le verbe français. Paris : Hachette/Larousse.

Fayol, M. \& Jaffré, J.-P. (2014). L'orthographe. Paris : Presses Universitaires de France.

Flumian, C., Labascoule, J. \& Royer, C. (2004). Rond-point 2. Barcelone/Saint-Martin-d'Hères : Difusión/Presses Universitaires de Grenoble.

Goes, J. (2015). « Comment sortir du Bled ? De la difficulté des natifs et des non-natifs à se distancier de la tradition grammaticale ». Le français dans le monde. Recherches et applications, $\mathrm{n}^{\circ} 57$. pp. 59-69.

Gombert, J.-E. (1990). Le développement métalinguistique. Paris : Presses Universitaires de France. 
Griggs, P. (2002). « À propos de l'effet de l'activité métalinguistique sur les processus de production en L2 ». In Cicurel, C. \& Véronique, D. (dir.). Discours, action et appropriation des langues. Paris : Presses Sorbonne Nouvelle. pp. 53-65.

Guédon, P. \& Poisson-Quinton, S. (2013). La grammaire du français en 40 leçons et 201 activités. B1. Paris : Éditions Maison des Langues.

Kaneman-Pougatch, M. ; Beacco di Giura, M. ; Trevisi, S. \& Jennepin, D. (1997). Café Crème 1. Méthode de français. Paris : Hachette Français langue étrangère.

Lavenne, C. \& Bérard, É. (2001). Studio 60-1. Méthode de français. Paris : Didier.

Martinet, A. (1960/2008). Éléments de linguistique générale. Paris : Armand Colin.

Neveu, F. (2004). Dictionnaire des sciences du langage. Paris : Armand Colin.

Pinchon, J. \& Couté, B. (1981). Le système verbal du français. Paris : Nathan.

Poisson-Quinton, S. (2014). La grammaire du français en 44 leçons et 230 activités. A1. Paris : Éditions Maison des Langues.

Surcouf, C. (2011). «L'enseignement et l'apprentissage de la conjugaison en FLE : comment réduire les difficultés engendrées par l'orthographe?». Tranel 54. pp. 91-110.

Surcouf, C. (2014a). « Résolvera-t-on l'énigme de la formation du Futur ? Réanalyse et origine possible des variantes non-normées de la morphologie du Futur ». Verbum 36-1 - Réanalyses, indétermination catégorielle et flou sémantique. pp. 35-54.

Surcouf, C. (2014b). "Epistemological tensions between linguistic description and ordinary speakers' intuitive knowledge: examples from French verb morphology". Carnets de grammaire 22. pp. 347-371.

\section{NOTES}

1. Les apprenants pouvaient les écouter à leur guise et individuellement à l'aide d'écouteurs sur leur propre téléphone portable.

2. L'orthographe française étant l'une des plus difficiles à apprendre et à utiliser (Fayol \& Jaffré, 2014 : 23).

3. La liste de ces ouvrages peut être obtenue auprès des auteurs.

4. Cependant Tempo 1 (Bérard et al., 1996: 191) et Studio 60-1 (Lavenne \& Bérard, 2001: 100) fournissent respectivement les terminaisons -rai, -ras, etc., et -erai, -eras, etc. pour les verbes du 1 er groupe.

5. Formes dont la décomposition est impossible à mettre en relation avec d'autres formes du paradigme de ces verbes.

6. Pour un examen plus détaillé et une réflexion épistémologique autour de ces options, voir Surcouf (2014a) et, de manière plus indirecte, Surcouf (2014b).

7. Le corpus peut être obtenu auprès des auteurs.

8. Nous n'a pas été utilisé en raison de la rareté de son usage à l'oral.

9. Le chiffre indique le groupe, les lettres l'apprenant.

10. La transcription phonétique reflète la formulation utilisée par l'apprenante, qui en l'occurrence semble utiliser l'Infinitif.

11. Pour travailler sur la langue parlée, il faut pouvoir se la représenter et la conserver en mémoire suffisamment longtemps. La mémoire à court terme ne permet pas d'en observer de longs fragments de façon fiable. [...] Pour étudier le parlé, nous devons donc nous en donner des représentations graphiques: 
par des transcriptions écrites et par des transpositions de la matière sonore. (Blanche-Benveniste, 2003 : 321).

12. Cela tend à prouver que les ouvrages de référence, qui alimentent le discours ordinaire de la grammaire, sont à l'origine de formations fautives.

\section{RÉSUMÉS}

Cet article examine le rôle du discours grammatical normatif véhiculé par les manuels et les grammaires de FLE - et fondé sur l'écrit - dans les activités de conceptualisation grammaticale. Nous avons observé 5 groupes de 4 apprenants de niveau B1 (en milieu universitaire homoglotte) lors d'une tâche collaborative de conceptualisation des règles de formation du Futur simple à partir d'un corpus oral enregistré. Les interactions engendrées par l'activité montrent que la majorité des participants raisonnent sur la base de l'écrit en dépit du caractère exclusivement oral des données fournies. Les étudiants tendent ainsi à prendre des notes en mobilisant des connaissances antérieures de formation du Futur sur la base de l'Infinitif écrit (jouer+a), règle pourtant incompatible avec la réalité de la morphologie orale dans la plupart des cas $(* / 3 \mathrm{we} /+/ \mathrm{a} /$ ). Cette tension entre l'écrit et l'oral, commune à tous les groupes observés, entraine des difficultés de conceptualisation du phénomène ciblé, et une certaine déstabilisation chez les apprenants. La démultiplication des commentaires métalinguistiques et métacognitifs révèle une intense activité réflexive, qui pourrait, à terme, permettre une déconstruction du discours de la grammaire ordinaire.

This article examines the role of normative grammatical explanations - conveyed by textbooks and grammars of French as a foreign language, and mainly based on writing - in grammatical conceptualization activities. We filmed 5 groups of 4 B1 learners listening to a recorded oral corpus, and engaged in a collaborative task of conceptualization of the formation rules of the "Futur simple". Interactions within the groups show that most participants base their reasoning on writing in spite of the oral data provided to them. While taking notes, students tend to assume -according to their previous grammatical knowledge- that "Futur simple" is constructed on the basis of the written form of the Infinitive (e.g. jouer $+a$ ), a rule which, in most cases, is inconsistent with spoken French morphology $(* / 3 w e /+/ a /)$. This tension between written and oral forms observed in all groups -, leads to conceptualization difficulties and a certain destabilization among learners. However metalinguistic and metacognitive comments reveal an intense reflexive activity, which could ultimately allow some deconstruction process of ordinary grammar explanations.

\section{INDEX}

Mots-clés : conceptualisation grammaticale, grammaire de l'oral, réflexion métalinguistique, grammaire normative, morphologie, futur simple

Keywords : grammatical conceptualization, oral grammar, metalinguistic reflection, normative grammar, morphology, futur simple 


\section{AUTEURS}

\section{ANICK GIROUD}

Université de Lausanne, Faculté des Lettres, École de Français Langue Étrangère Anick Giroud est maitre d'enseignement et de recherche à l'Ecole de Français Langue Etrangère de l'Université de Lausanne. Spécialiste en didactique du FLE, ses recherches portent actuellement sur l'appropriation de la grammaire du FLE en milieu guidé, dans ses aspects sociocognitifs.

Courriel : anick.giroud[a]unil.ch

\section{CHRISTIAN SURCOUF}

Université de Lausanne, Faculté des Lettres, École de Français Langue Étrangère Christian Surcouf est maitre d'enseignement et de recherche à l'université de Lausanne. Ses travaux portent notamment sur la sémantique de la temporalité en français, la morphologie verbale, la description de l'oral d'un point de vue à la fois épistémologique, linguistique et didactique. Depuis 2016, en collaboration avec Alain Ausoni, il travaille à la constitution d'une base de données orales (Florale) à des fins pédagogiques.

Courriel : christian.surcouf[a]unil.ch

\section{MALIKA BEN HARRAT}

Université de Lausanne, Faculté des Lettres, École de Français Langue Étrangère Malika Ben Harrat est enseignante de FLE et assistante diplômée à l'Ecole de Français Langue Etrangère de l'Université de Lausanne. Doctorante en didactique du FLS, ses recherches portent sur l'appropriation du français chez des migrants adultes. Elle s'intéresse aux influences sociales et psycho-affectives sur les trajectoires d'appropriation.

Courriel : malika.benharrat[a]unil.ch 